\title{
Gravitational Lensing Signature of Long Cosmic Strings
}

\author{
Andrew A. de Laix, ${ }^{1}$ Lawrence M. Krauss, ${ }^{1,2}$ and Tanmay Vachaspati ${ }^{1}$ \\ ${ }^{1}$ Physics Department, Case Western Reserve University, Cleveland, Ohio 44106-7079 \\ ${ }^{2}$ Theory Division, CERN, 1211 Geneva, Switzerland \\ (Received 3 February 1997; revised manuscript received 6 May 1997)
}

\begin{abstract}
The gravitational lensing by long, wiggly cosmic strings is shown to produce a large number of lensed images of a background source. In addition to pairs of images on either side of the string, a number of small images outline the string due to small-scale structure on the string. This image pattern could provide a highly distinctive signature of cosmic strings. Since the optical depth for multiple imaging of distant quasar sources by long strings may be comparable to that by galaxies, these image patterns should be clearly observable in the next generation of redshift surveys such as the Sloan digital sky survey. [S0031-9007(97)04026-X]
\end{abstract}

PACS numbers: 98.62.Sb, 98.80.Cq

An exciting outcome of the interplay between particle physics and cosmology is the realization that topological defects may be present in our Universe and may help to resolve some long-standing puzzles such as the origin of structure formation. A particular scenario which has been investigated over the past two decades is one where the relativistic motion of cosmic strings induces large-scale structure formation in the wakes that trail behind them [1]. Such strings would be present even now and, if observed, would be a culminating point for many exciting ideas in particle physics and cosmology. Past research has mostly focused on the observation of cosmic strings by the anisotropies they produce in the radiation background, and by the simple gravitational lensing characteristics of long straight strings. In this paper we reconsider this second approach and present a new signature in the lensing of galaxies or quasars resulting from realistic long cosmic strings. While the signature we describe is striking, because very few long strings are expected to span our horizon volume, one requires a survey over a significant fraction of the sky in order to place reliable constraints on cosmic string scenarios for the formation of large scale structure. The Sloan digital sky survey (SDDS) should be able to do just this.

Cosmic strings are lineal gravitating sources with tension along the string equal to the mass per unit length $\mu$. In the case of strings produced at the grand unified epoch (cosmic time $t \sim 10^{-35} \mathrm{sec}$ ), $\mu \sim 10^{22} \mathrm{gm} / \mathrm{cm}$. The gravitational effects of such strings are characterized by the dimensionless parameter $8 \pi G \mu \sim 10^{-5}$, implying they are strong enough gravitational sources to seed structure formation in the Universe. A string network consists of two distinct components: closed loops of string, and long (infinite) strings. Loops are typically of size $\leq \Gamma G \mu t \sim 10^{-4} t$ and live for about one Hubble time. $(\Gamma \sim 100$ is a numerical factor associated with the rate of gravitational radiation.) For infinite strings, numerical simulations of string networks show that there are order 10 long strings within a horizon volume at any epoch. These long strings sweep across the horizon at relativistic speeds and collide and reconnect with other strings. A very important fact, which has emerged after intensive numerical study of the string network evolution, is that these long strings are not straight, but have kinks and wiggles on them [2]. The characterization of this small-scale structure is not fully determined [3], especially since the string simulations do not yet include dissipation of string energy to gravitational radiation.

We should emphasize that the results given in the literature [4] describing the string network are ensemble averaged results. For gravitational lensing by long strings, however, it will turn out that the departures from the ensemble averaged string are all important. For example, even if the average string has fractal dimension close to 1 , the sharp discontinuities (kinks) on the string are vital to the gravitational lensing signature of strings. Unfortunately, no suitable characterization of such fluctuations is to be found in the current literature, so we were forced to generate our own ensemble of long strings. The initial string network is generated by laying down random phases on a periodic lattice using the method described by Vachaspati and Vilenkin [5]. Then we use the algorithm devised by Smith and Vilenkin [6] to evolve a network of strings in flat spacetime which allows us to generate a long string sample quickly and efficiently. (A detailed discussion of the numerical simulation will be presented in [7].) In using the flat spacetime evolved strings, our hope is that more realistic string ensembles will also yield qualitatively similar results.

Our simulations were run for a time equal to half the light crossing time of the box, and so the periodic boundary effects did not become significant. Segments of string within a cube with sides half the length of the box have had sufficient time to relax to a constant, fractal structure, and it is these segments which we use for lensing calculations since longer segments retain the random walk structure of the initial conditions.

After generating a long string, the next step is to find photon trajectories in its gravitational field. The location of the string at time $t$ is given by a vector function $f(\sigma, t)$ 
where $\sigma$ is a parameter along the string. In the absence of the strings, photons would travel from the source to the observer along the trajectory described by their constant initial four velocity $\gamma^{\mu}$. When a string is present, the photons are deflected, but, as $8 \pi G \mu$ is very small, we need only calculate the bending to first order in this parameter, that is, in the weak field approximation where we write the metric as $g_{\mu \nu}=\eta_{\mu \nu}+h_{\mu \nu}$. In the work on lensing by string loops [8], it was shown that for any compact lens, even those moving at relativistic speeds, the photon deflection can be reduced to a single integral along the string on the time slice: $t_{0}=f_{\|}\left(\sigma, t_{0}\right)$, where $f_{\|}$is the component of $\boldsymbol{f}$ that is parallel to the photon trajectory. To derive the deflection formula in [8], it was assumed that the source and observer reside at distances much larger than the size of the loop, but in the case of long strings, the source and observer are separated by about the horizon size, while the length of string which contributes to the gravitational potential is equally long. We can show, however, that for photons passing near the string, the dominant effect will be produced by only a small fraction of the overall string [7]. So let us consider the deflection arising from a finite segment of an infinite string over which $\sigma$ ranges from $\sigma_{1}$ to $\sigma_{2}$. In this case the deflection angle is

$$
\bar{\alpha}_{i}=-4 G \mu \int_{\sigma_{1}}^{\sigma_{2}} d \sigma\left[\frac{F_{\mu \nu}(\sigma, t) \gamma^{\mu} \gamma^{\nu}}{1-\dot{f}_{\|}} \frac{f_{\perp i}}{f_{\perp}^{2}}\right]_{t=t_{0}},
$$

where, $\quad \boldsymbol{f}(\sigma, t)=\boldsymbol{f}_{\perp}+\boldsymbol{f}_{\|}, \quad F_{\mu \nu}=\dot{f}_{\mu} \dot{f}_{\nu}-f_{\mu}^{\prime} f_{\nu}^{\prime}-$ $\eta_{\mu \nu} \dot{f}^{z}$, and, $f_{\perp}$ is the component of $\boldsymbol{f}$ which is perpendicular to the photon trajectory.

In Eq. (1) we have also discarded a boundary term whose effect on the image distortion is suppressed by the ratio of the size of a galaxy to the distance between the galaxy and the string. We assume that the galaxy is at a large distance from the string and are justified in ignoring this contribution to $\bar{\alpha}$.

To calculate the photon deflection in Eq. (1), we need to find the string coordinates on the time slice given by $t_{0}$. For this, we took the unperturbed photon trajectory to be parallel to the $z$ axis and found $f_{\|}$at all times using our long string evolution code. This allows us to numerically solve for $t_{0}$ and obtain the string trajectory at the particular time slice needed in Eq. (1). All our string sections had fractal dimension of about 1.25 within the scale of our 512 by 512 box and above the numerical cutoff scale $\Delta \sigma$ which we set to correspond to a physical scale given by $\Delta \sigma / D_{l}=0.1^{\prime \prime}$, where $D_{l}$ is the angular diameter distance measured from the observer to the string segment. This choice of $\Delta \sigma$ is small enough for discretization effects to be unimportant in our study of lensing and large enough that the string segment we have constructed stretches across a big enough patch of the sky. To minimize truncation errors, we consider lensing only in a $25 \operatorname{arcsec} \times 25$ arc sec subpatch.
To see how the deflection in Eq. (1) causes lensing, consider an axis connecting the observer to the lens, and suppose that there is an image located at a small angular displacement $\mathbf{x}$ from the axis. If we define $\boldsymbol{\alpha}=$ $\bar{\alpha}\left(D_{l s} / D_{s}\right)$, where $D_{l s}$ is the angular diameter distance from the lens to the source and $D_{s}$ is the angular diameter distance from the observer to the source, then the angular location of the source, $\mathbf{y}$, which would produce this image is given by $\mathbf{y}=\mathbf{x}-\boldsymbol{\alpha}(\mathbf{x})$, in the limit of small angles which is always valid here. We have also assumed the thin lens approximation in which the dimensions of the lens are much smaller than $D_{l s}$ or $D_{s}$. This is valid if we consider short segments of string compared to the horizon scale.

It is worthwhile to point out the length scales in the problem, considering two possible sources for lensing: quasars and high redshift galaxies. Quasars are very luminous, pointlike objects with typical redshifts of about $z \sim$ $2-5$. The typical angular separation of quasars is much larger than that of the expected image separation, so lensed pairs will be distinct. Another set of objects worth observing are galaxies at a redshift of $z \sim 2-3$, with the visible portion of such galaxies having angular diameters of roughly $0.5^{\prime \prime}$. The angular separation for the galaxies is about $10^{\prime \prime}$ [9]. The angular diameter of the length scale associated with small-scale structure on the string is $\Gamma G \mu t$, which, for a string at a redshift of $z \sim 1$, is very similar to the angular separation of high redshift galaxies corresponding to an angular scale $\sim 10^{\prime \prime}$ for $\Gamma=100$. Finally, note also that the mass associated with small scale string structure is $O\left(10 M_{\mathrm{gal}}\right)$, so that multiple lensed images could have separations on the order of $10^{\prime \prime}$, compared to multiple imaging by galaxies, which generally yields image separations of $1-3^{\prime \prime}$.

First we shall consider the lensing of a quasar due to a long cosmic string. In Fig. 1, we show string lensing for four different quasars each located at a redshift of $z=2$. The projected string configuration is located at $z=1$, and is shown by the dotted line, where only the contribution from the string shown was used in calculating the image locations. The hatched circle shows the location of the unlensed source, while the open circles show the locations of the various images, where the areas of the open circles are proportional to the magnification of the images relative to the source.

In Fig. 2 we show the same projected segment of string as used for the quasar lensing along with the unlensed images of seven circular sources located at $z=2$. The locations of these sources are chosen randomly in the plane and they have roughly the proper size and density to correspond to high redshift galaxies. In Figure 3 we see the images of these sources as they would appear to a terrestrial observer. ( $G \mu=10^{-6}$ in these simulations.) Note that the $0.1^{\prime \prime}$ resolution is consistent with that of the Hubble Space Telescope, while ground based telescopes achieve resolutions of about $0.5^{\prime \prime}$.

It is clear from Figs. 1 and 3 that a sequence of small demagnified images outline the string. One can 


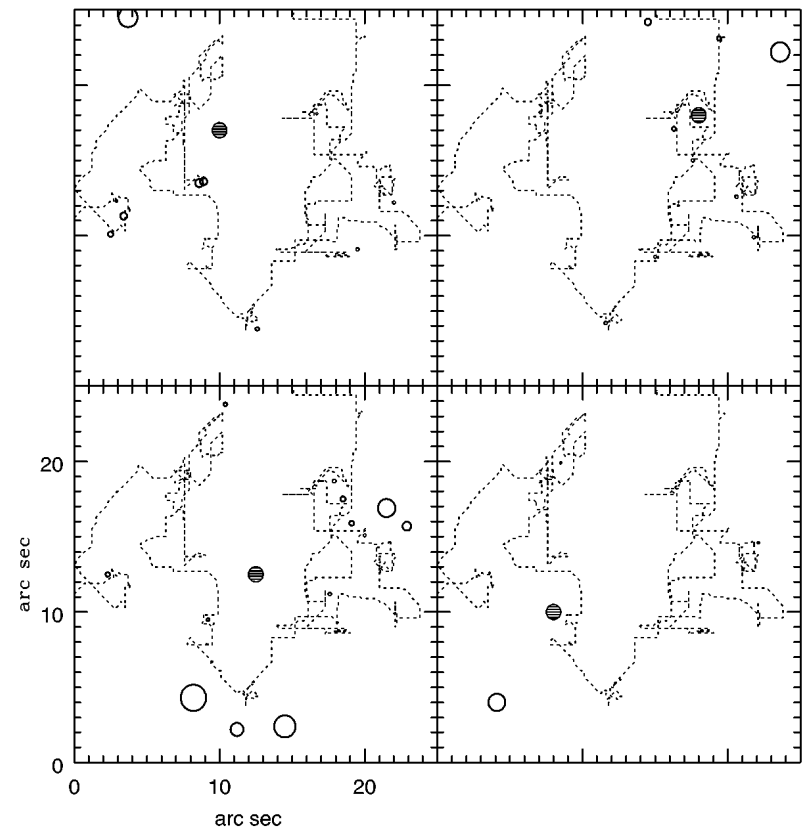

FIG. 1. Several quasar lensing systems. The hatched circle shows the location of the unlensed source and the open circles show the location of the resulting images produced by the string segment (dotted line). The ratio of the areas gives the relative magnification to the source.

qualitatively understand this result by considering a chain of point masses separated by distances less than their Einstein radius. Between the masses, the gravitational deflection can be canceled by the opposite attraction of the two masses. This allows the formation of a small image near the axis connecting the two masses, and for

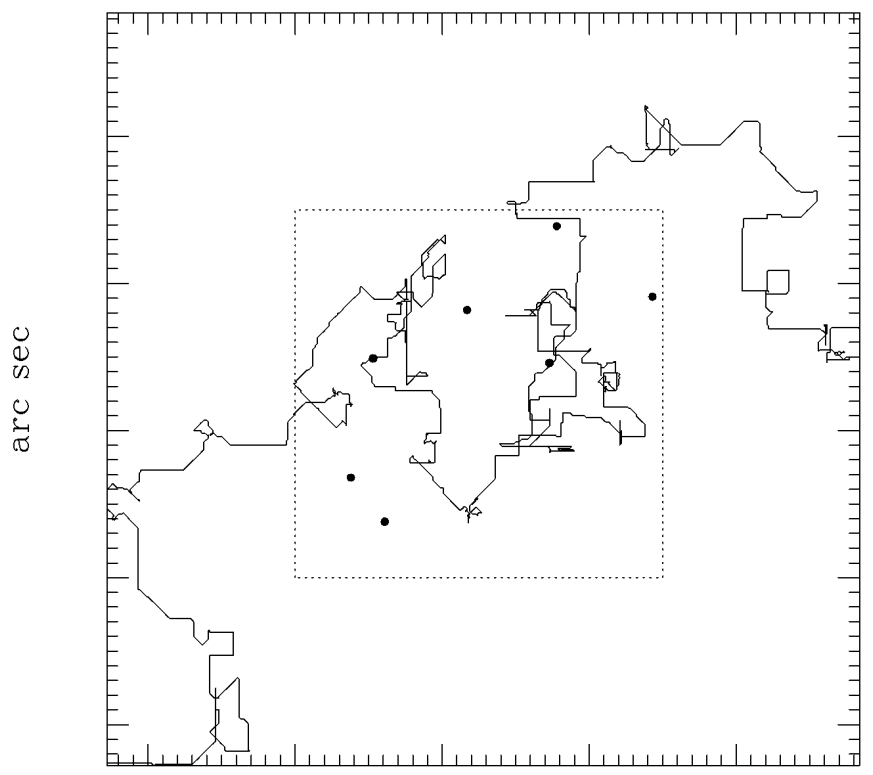

$\operatorname{arc~sec}$

FIG. 2. The projected string configuration along with the unlensed sources. Images were found for the region inside the dashed region. a chain, one might expect to see several small images. In the case of a string, it is the wiggles and kinks which provide the breaks needed to form the small images. The effect is similar to that observed for open string loops [8]; photons passing through a kink are subject to a diminished deflection which can allow an image to be formed.

Thus, the lensing pattern by strings has two distinct signatures: a sequence of baby images that outline the string and a set of major images that lie on either side of the string. The first feature depends on the frequency and magnitude of small-scale structure of the string and can be highly distinctive of the stringy nature of the lens. The second feature does not depend on the small-scale structure of the string and is similar to the previously considered lensing by straight strings due to the conical nature of spacetime that they produce.

It should be noted, however, that while the galaxy images in Fig. 3 are striking, it is not so clear that actual observed galaxy lensing by strings would be quite so distinctive. First, the galaxy brightnesses are much smaller, so that the small images near the string may not be visible. Second, due to the higher space density of galaxies, image separations due to string lensing will be comparable to the separations of galaxies themselves. For both reasons, we suggest that a survey involving quasar lensing is probably the most efficient first step in any attempt to probe for lensing due to cosmic strings.

With that in mind, we consider the probability of observing lensing by long cosmic strings and strategies for observation. As described earlier, it is most useful to first consider surveys of objects which are widely separated on arc sec scales, such as quasars. A recent probability

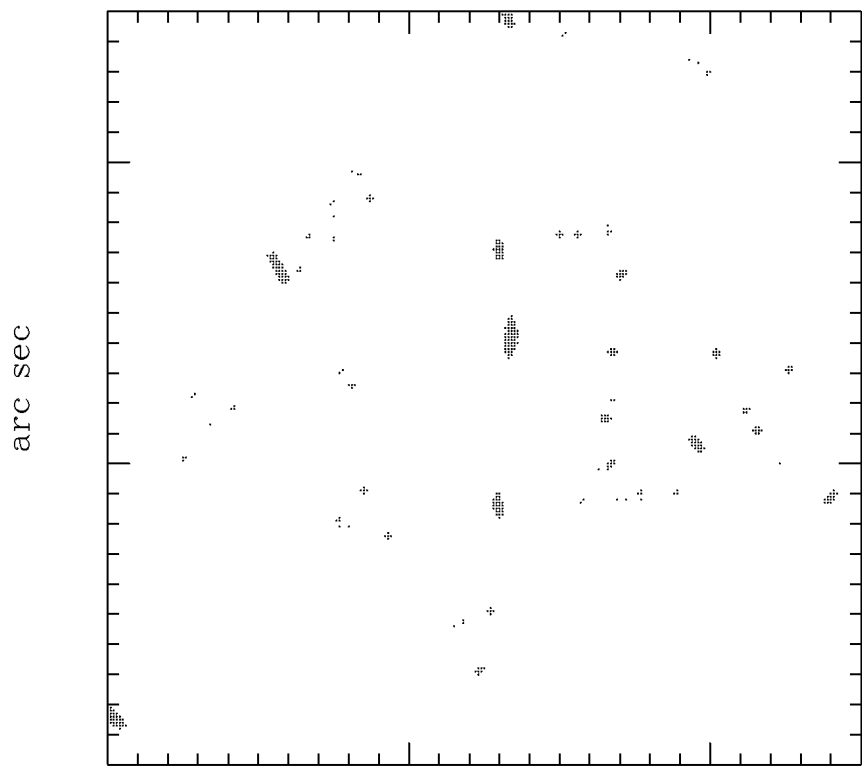

$\operatorname{arc~sec}$

FIG. 3. The resulting images of the sources shown in Fig. 2. The full string shown in Fig. 2 were used in determining the lensing effects. 
calculation, based on estimates of the long string density arising from numerical simulations [4], and also on the projected angular cross section for string lensing yields an optical depth of $\tau \approx(0.2-0.6) \times 10^{-3} G \mu_{6}$ for $z=$ $2-3$, where $G \mu_{6}=G \mu \times 10^{6}$ (see [7] for further details). To turn this into an actual prediction of lensing frequency in any specific survey requires an analysis of such issues as selection effects, etc. [10]. However, it is useful to compare this optical depth to that due to lensing of high redshift quasars by the known galaxy population. In the ratio of these quantities many such effects should cancel. For a flat universe, the optical depth, assuming the dominant lensing is by elliptical galaxies modeled as isothermal spheres, is $\approx(2-4) \times 10^{-3}[10,11]$ in the same range. If account is taken of possible finite core effects [12], this number could be reduced by a factor of 2. Hence, the ratio of optical depths for string lensing to galaxy lensing in any large sample of quasars is roughly $0.1 G \mu_{6}$ to $0.5 G \mu_{6}$, suggesting a measurable fraction of all multiply imaged quasars would result from cosmic string lenses.

Because existing surveys have so far unearthed only on the order of 12 multiply imaged quasars, one cannot yet use them to place significant constraints on the parameters of long cosmic strings. In particular, because the long string density in our horizon volume is small, a significant fraction of the sky would have to be surveyed before a definitive constraint could be derived (i.e., the string sample is non-Gaussian). A good search strategy would be to observe a large number of quasars in a wide angular field, and the Sloan digital sky survey is precisely such an observation. Approximately $10^{5}$ quasars will be observed over $1 / 4$ of the sky with typical redshifts of about $z \sim 2$. One can expect that the SDSS will observe at least on the order of several hundred lensed quasars, with at least roughly $10 \%$ of that number due to long strings, if they exist in the sample volume. In advance of detailed figures on selection effects, etc., once the sample is obtained it is difficult to quote an exact bound which might be expected.

However, we can estimate what kind of limits might be achievable in principle. Approximating a cosmic string network as an ensemble of uncorrelated links which, based on numerical simulations, are approximately $10 \%$ of the horizon length, the variance in the predicted string distribution in a sample can then be straightforwardly calculated using Poisson statistics. Fluctuations arise from the number and distribution of quasars and the variations in the number of string segments. To this we must add in quadrature a variance associated with the theoretical uncertainty in the mean string density, about $20 \%$. Assuming, for example, that all of the quasars reside at a redshift $z=2$ (a reasonable assumption for SDSS), if one expects $N_{q}$ quasars and $N_{s}$ string segments to be observed, then the observed number of lenses is $N_{q} \tau \pm \sqrt{N_{q} \tau+\left(N_{q} \tau\right)^{2} / N_{s}+\left(0.02 N_{q} \tau\right)^{2}}$. For $G \mu_{6}=$ 1, SDSS will observe $N_{q}=10^{5}$ quasars while there should be roughly $N_{s}=100$ independent string segments. Using $\tau=2 \times 10^{-4}$, the $99 \%$ lower bound is 1 observed lens, so that a nondetection would rule out strings with tensions $G \mu>10^{-6}$ at the three $\sigma$ limit. Since current surveys cover a very small solid angle, while the predicted number of observed string induced lenses is approximately $1 G \mu_{6}$, the variance is high, so only tensions greater than $G \mu_{6}>O(10)$, are ruled out at the $95 \%$ confidence level, a limit weaker than other current limits.

Of course in a crowded field it may be difficult to initially distinguish the string signature. For this reason, we are attempting to build an archive of artificially lensed images of real galaxy fields in order to explore this issue in greater detail [13]. It is worth emphasizing that only a structure which acts like a filament of mass points will induce the lensing structures described here, so in principle the string lensing signature is unique. Thus, while it may be difficult to initially uncover the string signal, it is unlikely to obtain false positives.

Here is where galaxy observations can also be useful in attempting to distinguish the string signal. By using a telescope such as the Hubble Space Telescope, one can observe the high redshift galaxies in the neighborhood of the lensed quasar, looking for string lensing of galaxies. Together with the quasar images, these would provide firmer evidence of cosmic strings, or by their absence, rule them out as seeds of structure formation.

We thank Glenn Starkman and Heather Morrison for discussions, and the Department of Energy for support.

[1] A. Vilenkin and E.P.S. Shellard, Cosmic Strings and Other Topological Defects (Cambridge University Press, Cambridge, 1994).

[2] D. P. Bennett and F. R. Bouchet, Phys. Rev. D 41, 2048 (1990); B. Allen and E. P. S. Shellard, Phys. Rev. Lett. 64, 119 (1990).

[3] For example, see G. R. Vincent, M. Hindmarsh, and M. Sakellariadou, SUSX-TH-96-020; Report No. astro-ph/ 9612135.

[4] See the papers by D.P. Bennet, F. R. Bouchet, N. Turok, A. Albrecht, E. P. S. Shellard, and B. Allen, in The Formation and Evolution of Cosmic Strings, edited by G.W. Gibbons, S. W. Hawking, and T. Vachaspati (Cambridge University Press, Cambridge, 1990).

[5] T. Vachaspati and A. Vilenkin, Phys. Rev. D 30, 2036 (1984).

[6] A. G. Smith and A. Vilenkin, Phys. Rev. D 36, 990 (1987).

[7] A. A. de Laix Phys. Rev. D (to be published).

[8] A. A. de Laix and T. Vachaspati, Phys. Rev. D 54, 4780 (1996).

[9] M. J. Sawicki, H. Lin, and H. K. C. Yee, astroph/9610100.

[10] E. L. Turner, J. P. Ostriker, and J. R. Gott, Astrophys. J. 284, 1 (1984).

[11] L. M. Krauss and M. White, Astrophys. J. 394, 385 (1992).

[12] G. Hinshaw and L. M. Krauss, Astrophys. J. 320, 468 (1987).

[13] See http://theory2.phys.cwru.edu/ delaix/cosmic.html . 